\title{
PHOTONIC BAND GAP ACCELERATOR CAVITY DESIGN AT 90 GHz*
}

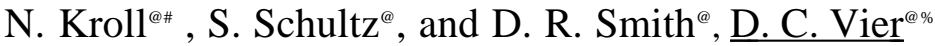

\begin{abstract}
In previous work we have proposed utilizing Photonic Band Gap (PBG) structures as a new class of high-energy, high-intensity accelerator cavities. We have completed extensive MAFIA numerical calculations of multicell PBG structures, with each cell consisting of a square array of metal cylinders terminated by conducting sheets and surrounded by microwave absorber on the periphery. We find that our PBG structure has good higher order mode suppression, superior pumping properties, and potential fabricational advantages when compared to conventional cavity designs. These advantages scale favorably with increasing frequency, leading us to propose use of a PBG accelerator structure at W-band $(91.392 \mathrm{GHz})$.
\end{abstract}

\section{INTRODUCTION}

We have previously proposed that a new type of microwave resonator, the Photonic Band Gap (PBG) cavity, may be useful as a novel high energy accelerator structure [1]. Briefly, the PBG cavity structure consists of a $2 \mathrm{D}$ periodic lattice of metal cylindrical scatterers, which are bound between a pair of metal plates. Removing a cylinder from the center of the lattice forms a defect site, where a highly localized electro-magnetic accelerating "defect" mode can exist over a wide range of lattice parameters.

When the frequency of the "defect" mode lies within the bandgap of the PBG structure the energy density decays exponentially in all directions from the defect site. This rate of energy decay can be very large, typically two orders of magnitude per lattice constant. At room temperature, with $\mathrm{Q}$ values corresponding to a copper construction, a $7 \times 7$ lattice of cylinders easily suffices to prevent any significant energy loss of the fundamental in the radial direction.

In high energy accelerators Higher Order Modes (HOMs) such as dipole modes, can be excited by off-axis electrons and other perturbations. In a usual pillbox cavity these HOMs, unless specially damped (e. g. by damped detuning), have $\mathrm{Q}$ values comparable to the fundamental mode, and can generate a long-lived wakefield that will seriously degrade the next electrons in a traveling bunch.

*Work supported by the U.S. Department of Energy under Contract Nos. DOE-DEFG-03-93ER40793 and DOE-DEFG-0393ER40759

${ }^{\circledR}$ Department of Physics, University of California, San Diego, 9500 Gilman Drive, La Jolla, CA 92093-0319

${ }^{\text {\#} S t a n f o r d ~ L i n e a r ~ A c c e l e r a t o r ~ C e n t e r, ~ S t a n f o r d ~ U n i v e r s i t y ~ S t a n f o r d, ~ C A ~}$ 94309

\% Email: dvier@ucsd.edu
The PBG cavity structure is novel in that the fundamental resonant mode can be strongly localized, with high $\mathrm{Q}$ and arbitrarily low leakage, while all other HOMs in the system can be very strongly damped. The way that this can be achieved for a PBG system, is to have the metal plates and cylinders surrounded by a very lossy absorbing material strategically placed around the periphery of the finite PBG lattice. It then becomes possible to have a suitably high $\mathrm{Q}$ for the fundamental mode, and simultaneously have all HOMs strongly damped. This condition can occur in a PBG system by a proper choice of parameters causing the HOMs to occur in "pass bands", and hence readily propagate to the periphery, where they may be absorbed. In contrast, the fundamental mode is highly localized within the center of the structure, and since there is no significant field amplitude at the periphery it is essentially undamped.

We have previously described the results of wakefield calculations for single PBG cavities without a beam iris [2]. In this paper we present results of extensive numerical calculations for an iris-coupled periodic PBG cavity structure. An iris in each metal plate, centered on the defect position axis, provides the coupling between successive cells, and as usual is the path of the pulsed electron beam [3]. We utilize MAFIA, a commercial finite-difference electromagnetic analysis program, to perform both frequency- and time-domain studies. We have concentrated on simulating the $\mathrm{Q}$ and loss-factors associated with the HOMs of the metal PBG cavity as a function of absorber geometry, and estimating the wakefield associated with the iris coupled chain of PBG cavities.

\section{HOM AND WAKEFIELD STUDIES IN THE FREQUENCY-DOMAIN}

Our main goal was to perform a complete calculation of the wakefield for a multicell PBG structure and to thoroughly test the hypothesis that all HOMs could be effectively damped by the peripheral absorber. To facilitate the wakefield calculation, we model our PBG cell as one of an inifinitely periodic set of identically coupled cavities. We choose the height of our cell $(34.99 \mathrm{~mm}$, including plate thickness) to set the frequency of the fundamental accelerating mode at $2.856 \mathrm{GHz}$, with $2 \pi / 3$ phase advance across the cell. The post diameter is 16.09 $\mathrm{mm}$, the lattice spacing is $42.9 \mathrm{~mm}$, the thickness of the plates is $6.0268 \mathrm{~mm}$, and the beam iris radius is $18 \mathrm{~mm}$. In Figure 1 we present the dispersion curves for the first seventeen (of the 160 actually calculated) dipole modes in the metal PBG cavity. 


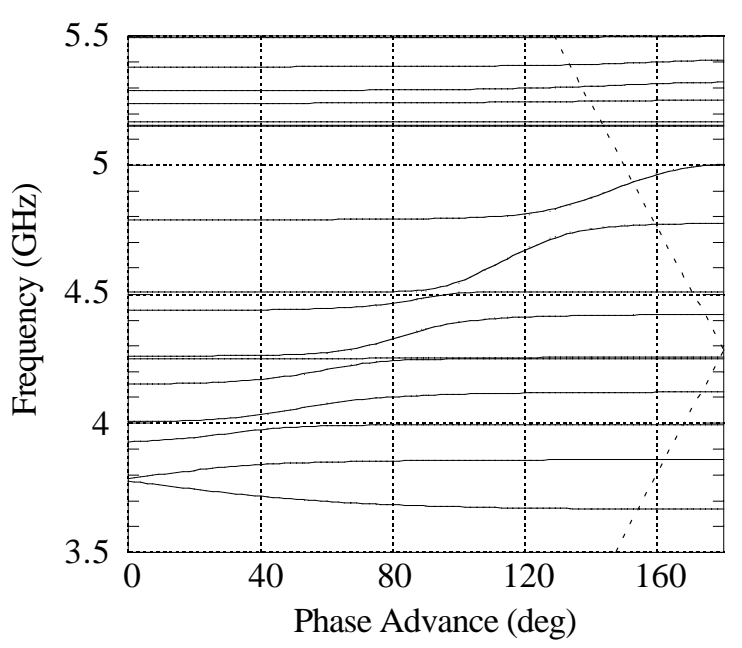

Figure 1. Dispersion curves for the first 17 dipole mode bands of our $7 \times 7$ PBG cavity as calculated by MAFIA. The intersection of each curve with the $\mathrm{v}=\mathrm{c}$ line (dashed) gives the synchronous phase and frequency for which each dipole mode kick-factor is calculated.

We are only concerned with modes whose frequency coincides with the synchronous frequency, since these may produce unwanted beam deflection if their kick-factor is large enough.

From the dispersion curves we determine the synchronous phase and frequency for each of the 160 calculated modes and then calculate the kick-factor for each of these synchronous modes. The resulting transverse wake potential can then be calculated from the formula:

$$
\text { Wake Potential }(t)=2 \sum_{n=1}^{N} V\left(\omega_{n}\right) \sin \left(\omega_{n} t\right)
$$

where $\mathrm{V}$ is the kick-factor, $\omega_{\mathrm{n}}$ is the frequency of the nth dipole mode, and $\mathrm{t}$ is the time step.

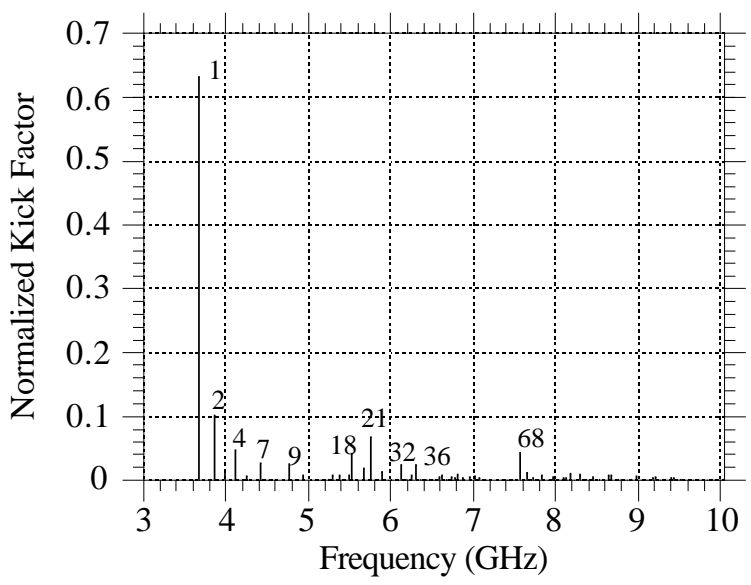

Figure 2. The synchronous kick-factors for the first 160 dipole modes $(0-10 \mathrm{GHz})$. The ten modes with the largest kick-factors are labeled with their respective mode number.
In Figure 2 the kick-factors, $\mathrm{V}\left(\omega_{\mathrm{n}}\right)$, normalized to the kick-factor of the first dipole mode of the equivalent pillbox cavity are shown for the lowest 160 synchronous modes. Most of the synchronous modes have negligible kick-factor, and we find that the wake potential calculated from the ten modes with the largest kick-factor (labeled in Figure 2 with their corresponding mode number) is nearly identical to that calculated using all 160 modes. The wake potential is found to be about half of that calculated for a pill-box cavity over the same frequency range $(0-10 \mathrm{GHz})$.

The most troublesome PBG dipole mode we found is the first (lowest) dipole mode. It has the largest contribution to the wake potential and is also found to be quasi-localized. All other synchronous PBG dipole modes with significant kick-factors were found to be highly extended in space. To obtain some idea of the effect of the beam iris parameters on this dipole mode, we calculated the kick-factor as a function of the beam iris radius (0-30 $\mathrm{mm}$ ) for both the lowest PBG and lowest pill-box dipole modes. The PBG mode was found to always have a lower kick-factor than the pill-box mode. The results also indicate that, with a beam iris radius of $18 \mathrm{~mm}$, our current PBG design is not optimal from the standpoint of minimizing the first dipole mode kick-factor. They suggest that improvement may be achieved for applications that can withstand a significant alteration in the beam iris radius. We have also determined that the effect of the beam iris thickness on the kick-factor of the first PBG dipole mode is minor.

\section{DAMPED WAKEFIELD STUDIES IN THE TIME-DOMAIN}

Of primary interest is the behavior of the metal PBG cavity when absorber is introduced around the structure. As the first PBG dipole mode is the only quasi-localized dipole HOM with a significant kick-factor, we expended serious effort in designing an absorber configuration that would significantly damp this mode without affecting the $\mathrm{Q}$ of the fundamental mode. The three dimensional cone absorber configuration shown in Figure 3 was found to give optimal damping of the first PBG dipole mode, while minimizing the damping of the fundamental mode. The absorber dielectric parameters are $\varepsilon=27$ and $\tan (\delta)=0.23$, typical of the absorber material used at SLAC.

Utilizing MAFIA in the time-domain, the Q values for the ten dipole modes with largest kick-factors (Figure 2) were calculated in the presence of the absorber configuration shown in Figure 3. These dipole HOMs, including the first dipole mode, were all determined to have $Q$ values of $\sim 100$ or less, whereas the fundamental mode $\mathrm{Q}$ was only reduced by a few percent (from 11,950 to 11,540$)$. We note that the calculated $Q$ values of the dipole HOMs are only approximate, as the MAFIA timedomain solver does not usually allow us to perform timedomain simulations at the correct synchronous phases of each mode. 


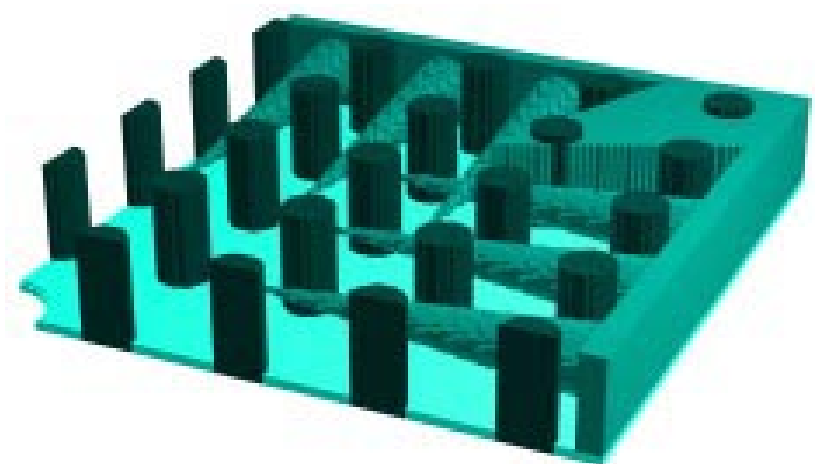

Figure 3. The absorber configuration found to be optimal for damping the lowest dipole HOM .

From the calculated dipole HOM Q values, we can compute a reasonable simulation of the wake potential for the PBG cavity in the presence of absorber by adding a Q time-dependence for each mode into the wake potential formula:

$$
\text { Wake Potential }(t)=2 \sum_{n=1}^{N} V\left(\omega_{n}\right) \sin \left(\omega_{n} t\right) e^{\frac{-\omega_{n} t}{2 Q}}
$$

In Figure 4 we plot the envelope of Eq. 2, the wake envelope function, for the ten damped PBG dipole modes with the largest kick-factor.

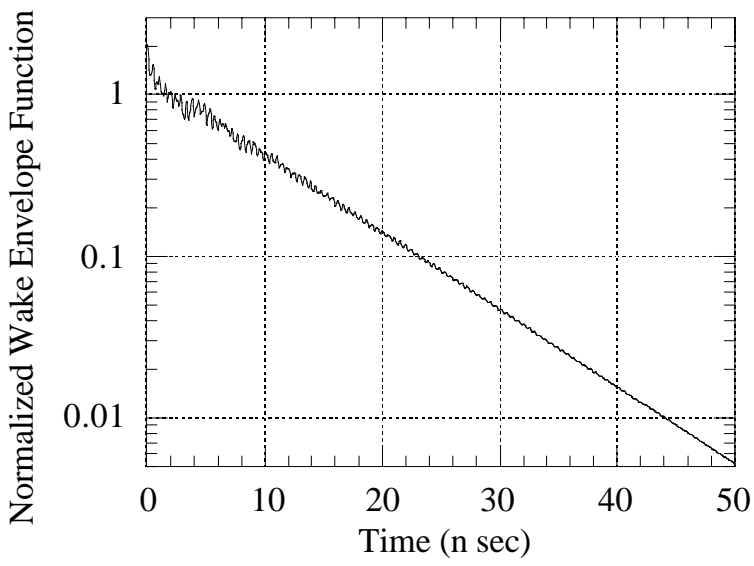

Figure 4. The calculated wake envelope function, with damping, for the ten dipole modes having the largest kickfactors. Note the semi-log scaling.

The longitudinal wake potential is also of interest for accelerator design, and we have utilized the same procedure as for the transverse wake potential (over the frequency range from $0-8 \mathrm{GHz}$ ). The simulation revealed that three monopole HOMs had a significant synchronous longitudinal kick-factor. Two are readily damped by the absorber configuration of Figure 3. However, the third monopole HOM (synchronous frequency=5.87 GHz) exhibited a degree of localization much greater than that of any of the dipole HOMs studied previously. This mode had been discovered in our earlier work, and is associated with a pseudo-PBG in the pass bands. For this undesired monopole HOM, we found it possible to perturb the inner cylinder configuration positions, such that its localization is significantly reduced (making the mode more easily damped), while the fundamental mode suffers only a minor perturbation.

In fact, the fundamental mode is extremely robust with respect to deviations of the innermost posts from a perfect lattice. Frequency deviations of the fundamental are of course expected, but can always be compensated for by appropriate changes in the lattice constant. What is most important is that there are no significant perturbations of the field patterns and degree of localization of the fundamental mode. This field pattern stability is due to the fundamental frequency occuring deep within a large PBG, where slight perturbations only act to increase or decrease the mode localization in a minor way. A given perturbation can however have a drastic impact on HOMs in the pass bands, effectively moving them from pseudolocalized to extended in space.

\section{FUTURE WORK}

The modes for the intrinsic PBG structure (i.e., without absorber) scale with frequency in the same manner as a pill-box cavity, or any other metal structure. Thus, all of the field properties, localization, etc. apply equally to a reduced size $1 / 32$ structure whose fundamental mode is at $91.392 \mathrm{GHz}$. The characteristic physical dimensions (in $\mathrm{mm}$ ) at $91.392 \mathrm{GHz}$ are: plate spacing $1.093 \mathrm{~mm}$, plate thickness $0.189 \mathrm{~mm}$, post diameter $0.504 \mathrm{~mm}$, and lattice constant $1.344 \mathrm{~mm}$. We believe that the fabrication of a multicell PBG structure based on components with these dimensions is practical, and should require only wellestablished NC and EDM machining techniques. The relatively open design of the PBG structure should allow for adequate pumping properties, as well as simplifying the tuning of multicell PBG structures operating at $91.392 \mathrm{GHz}$. A multicell test structure is under development.

\section{REFERENCES}

[1] D. R. Smith, Derun Li, D. C. Vier, N. Kroll, S. Schultz, "Recent progress on photonic band gap accelerator cavities", Proceedings of the seventh workshop on advanced accelerator concepts, Lake Tahoe, CA, October (1996)

[2] Derun Li, N. Kroll, D. R. Smith, S. Schultz, "Wake-field studies on photonic band gap accelerator cavities", Proceedings of the seventh workshop on advanced accelerator concepts, Lake Tahoe, CA, October (1996)

[3] N. Kroll, D. Smith, S. Schultz, "Photonic band-gap structures: a new approach to accelerator cavities", Third Advanced Accelerator Concepts Workshop, Port Jefferson, New York., June 1992. 